\title{
Generalização por 'reforço' ou ‘contraste’ no tratamento do desvio fonológico
}

\author{
Generalization by 'reinforcement' or 'contrast' in the treatment of \\ phonological disorders
}

\author{
Márcia Keske-Soares ${ }^{1}$, Gabriele Donicht ${ }^{2}$, Mardônia Alves Checalin³, Maria Rita Leal Ghisleniं ${ }^{4}$
}

\begin{abstract}
RESUMO
O objetivo deste estudo foi verificar as mudanças fonológicas obtidas pelo tratamento com o Modelo de Oposições Máximas Modificado, utilizando a abordagem do 'reforço' ou 'contraste', em sujeitos com diferentes graus de severidade do desvio fonológico (DF). Três sujeitos com DF, do sexo masculino, com idade média de seis anos e diferentes graus de severidade de DF, foram submetidos às avaliações fonoaudiológicas, incluindo a fonológica, e exames complementares. Posteriormente, foram submetidos à terapia fonológica pelo modelo de Oposições Máximas Modificado, considerando-se a abordagem do 'reforço' ou 'contraste' de traços distintivos e verificadas as seguintes generalizações: para itens não utilizados no tratamento; para outra posição na palavra; dentro de uma mesma classe de sons; e para outra classe de sons. O inventário fonético dos três sujeitos estava completo após a terapia; porém, o sistema fonológico estava incompleto para todos os sujeitos tratados. O S1 tratado, utilizando contraste com [dors/cor] e [ \pm cont], após tratamento permaneceu com dificuldade com [dors] e [-voc]. O S2 tratado pelo reforço com o [+voz], após tratamento persistiu com dificuldade com [+voz]. O S3 tratado pelo reforço com [+soant], [-voc], [+voz] e [cor/+ant], apresentou pós-tratamento dificuldade apenas com [-voc]. Após o processo terapêutico, observou-se evolução para todos os tipos de generalização estrutural analisados. Verificou-se que tanto os sujeitos tratados pelo 'reforço', quanto o tratado pelo 'contraste' apresentaram generalizações, não podendo diferenciar qual abordagem foi mais eficaz no tratamento do DF.
\end{abstract}

Descritores: Fala; Transtornos da articulação; Linguagem; Criança

\section{INTRODUÇÃO}

O desenvolvimento da fonologia faz parte do processo de aquisição da linguagem e se dá de forma gradativa e não-linear, entre o nascimento e os cinco anos de idade, pois nesse período ocorre o estabelecimento do sistema fonológico semelhante ao alvo-adulto. Crianças com Desvio Fonológico (DF) apresentam um desvio ou afastamento do componente fonológico da linguagem, já que existe um sistema, embora inadequado ${ }^{(1)}$.

Trabalho realizado no Curso de Fonoaudiologia do Centro de Ciências da Saúde da Universidade Federal de Santa Maria - UFSM - Santa Maria (RS), Brasil.

(1) Doutora, Professora do Curso de Fonoaudiologia e do Curso de Mestrado em Distúrbios da Comunicação Humana da Universidade Federal de Santa Maria - UFSM - Santa Maria (RS), Brasil

(2) Pós-graduanda em Distúrbios da Comunicação Humana da Universidade Federal de Santa Maria - UFSM - Santa Maria (RS), Brasil.

(3) Pós-graduanda em Distúrbios da Comunicação Humana da Universidade Federal de Santa Maria - UFSM - Santa Maria (RS), Brasil.

(4) Pós-graduanda em Distúrbios da Comunicação Humana da Universidade Federal de Santa Maria - UFSM - Santa Maria (RS), Brasil.

Endereço para correspondência: Maria Rita Leal Ghisleni. R. Sete de Setembro, 306, Perpétuo Socorro, Santa Maria - RS, CEP 97045-450. E-mail: ritaghisleni@yahoo.com.br

Recebido em: 21/8/2007; Aceito em: 29/8/2008
O DF pode ser classificado quantitativamente a partir do cálculo do Percentual de Consoantes Corretas (PCC) em diferentes graus de severidade: Severo; Moderado-Severo; Médio-Moderado e Médio ${ }^{(2)}$. Após a classificação do grau do DF, escolhe-se o modelo terapêutico, o qual promoverá o maior número de aquisições ao sistema fonológico da criança.

Existem diferentes abordagens terapêuticas para o tratamento dos DF. Dentre elas tem-se o Modelo de Oposições Máximas Modificado ${ }^{(3)}$, baseado no Modelo de Oposições Máximas $^{(4)}$. O objetivo deste modelo é promover a reorganização do sistema fonológico por meio da percepção auditiva, imitação da produção e produção espontânea dos sons-alvo, visando generalização e melhor inteligibilidade da fala.

Diversas pesquisas ${ }^{(5-8)}$ verificaram sucesso terapêutico por meio da ocorrência de generalização e aumento do número de sons do sistema fonológico de crianças com DF falantes do Português Brasileiro, submetidas à terapia pelo Modelo de Oposições Máximas.

Em relato de caso(6) comparando dois diferentes modelos terapêuticos, aplicados em duas crianças com DF, os seguintes tipos de generalização foram estudados e analisados: itens não utilizados no tratamento, para outra posição na palavra, dentro de uma mesma classe de sons e para outra classe de 
sons. Foram verificadas generalizações de todos os tipos, à exceção da generalização para outra classe de sons.

Em estudo ${ }^{(8)}$ com oito sujeitos com diferentes graus de DF, tratados pelo Modelo de Oposições Máximas Modificado ${ }^{(3)}$, foram analisadas as mudanças fonológicas e proposta uma nova abordagem de seleção dos alvos de tratamento, considerando a apresentação dos traços distintivos se por meio do 'contraste' ou do 'reforço'. A primeira considera o uso de dois segmentos-alvo que possuem valores opostos para o mesmo traço, ou seja, o trabalho de um traço menos marcado com um mais marcado; enquanto a segunda, considera o uso de segmentos-alvo com valores idênticos para o mesmo traço. Foi verificada mudança fonológica entre os sujeitos tratados pelo 'contraste' e os sujeitos tratados pelo 'reforço'.

As propostas terapêuticas baseadas em oposição de traços têm sido bastante estudadas. Um estudo comprova a eficácia no tratamento do DF por meio do 'contraste' ou do 'reforço' do traço a ser estimulado visando à generalização $0^{(8)}$; entretanto, não se sabe qual abordagem é mais eficaz.

A abordagem que se refere ao tratamento salientando o 'contraste' ou o 'reforço' dos traços, entretanto, ainda é pouco explorada. Portanto, o objetivo deste trabalho foi verificar as mudanças fonológicas obtidas pelo tratamento com o Modelo de Oposições Máximas Modificado ${ }^{(3)}$, utilizando a abordagem do 'reforço' ou 'contraste', em sujeitos com diferentes graus de severidade do desvio fonológico (DF).

\section{APRESENTAÇÃO DOS CASOS CLÍNICOS}

O presente trabalho trata-se de estudo de casos de sujeitos selecionados no banco de dados pertencentes a projeto aprovado no Comitê de Ética em Pesquisa da Universidade Federal de Santa Maria sob n ${ }^{\circ}$ 046/02.

Os sujeitos selecionados passaram por triagem, avaliação e terapia fonoaudiológica pelo Modelo de Oposições Máximas Modificado $^{(3)}$ no Serviço de Atendimento Fonoaudiológico (SAF) da Universidade Federal de Santa Maria. Para a realização desse estudo, os respectivos responsáveis assinaram o Termo de Consentimento Livre Esclarecido, autorizando a participação dos sujeitos no projeto.

Foram incluídas no estudo crianças do sexo masculino com DF, na faixa etária de seis anos, com diferentes graus de severidade do DF, tratados utilizando como estímulo pares maximamente opostos, com nível 2 na hierarquia ${ }^{(9)}$, ou seja, foram selecionados dois fonemas novos, com diferença de classe não-principal e com distinção máxima de traços, ou um fonema novo, com distinção de traços de classe principal e com distinção máxima de traços.

O diagnóstico de DF foi obtido após a realização das seguintes avaliações: Avaliação Fonológica da Criança $\left(\mathrm{AFC}^{(10)}\right.$; sistema estomatognático; exame articulatório; linguagem; vocabulário; psicomotricidade; discriminação auditiva; consciência fonológica; memória de trabalho e processamento auditivo simplificado. Avaliações complementares como audiológica, otorrinolaringológica e neurológica também foram consideradas para a seleção da amostra.

Após a aplicação da AFC foram realizadas as seguintes análises: contrastiva e por traços distintivos. Os resultados da análise contrastiva permitiram determinar se os fonemas estavam ou não adquiridos no sistema fonológico. Para tanto, foram considerados os seguintes critérios: $80 \%$ ou mais - som adquirido (SA); $79 \%$ a $40 \%$ - som parcialmente adquirido (SPA); $39 \%$ a $0 \%$ - som não-adquirido (SNA) ${ }^{(11)}$. Os resultados para a análise por traços distintivos foram obtidos por intermédio da verificação das substituições realizadas na análise contrastiva, considerando uma ocorrência mínima de 10\% das possibilidades $^{(12)}$.

Foram selecionados do banco de dados três sujeitos que apresentavam DMS (S1), DMM (S2) e DM (S3). Não foi selecionado um sujeito com DS, pois não foi encontrado no banco de dados um sujeito com DS do sexo masculino, na faixa etária de seis anos.

Os sujeitos selecionados foram tratados pelo Modelo de Oposições Máximas Modificado ${ }^{(3)}$ 'sendo realizadas duas sessões semanais de 45 minutos cada. Neste modelo de tratamento, primeiramente foi aplicada a linha de base (LB), uma sondagem dos sons que não se encontravam totalmente adquiridos no sistema fonológico. A LB foi realizada antes do início da terapia fonológica, na qual cada som foi testado a partir da nomeação de seis figuras, sendo utilizada para avaliar o progresso terapêutico posterior.

As sessões terapêuticas eram iniciadas com o bombardeio auditivo composto por, no mínimo, 16 palavras (oito com cada som-alvo). A estimulação da produção correta dos pares mínimos ocorria em duas etapas imitação e produção espontânea. Na primeira, a criança deveria produzir os alvos após o modelo do terapeuta, devendo atingir um percentual de $80 \%$ ou mais de produções corretas, para passar, então, à fase de produção espontânea. Nesta etapa, os alvos deveriam ser produzidos sem o modelo do terapeuta. Ao final de cada sessão, calculava-se o percentual de produções corretas da criança e, também, era lido novamente o bombardeio auditivo. Além disso, orientavam-se os responsáveis à leitura do bombardeio auditivo e cópias das figuras enfocadas em terapia.

Após o tratamento, os resultados da avaliação final (AF) foram verificados para S1 e S2, por meio da sondagem após 20 sessões de terapia, e para S3, por meio do AFC após 10 sessões de terapia. Desse modo, para os S1 e S2, analisou-se o total de 22 sessões, sendo uma sessão de LB, 20 sessões de tratamento e uma sessão de AF. E para S3, analisou-se um total de 12 sessões, sendo uma sessão AI, 10 sessões de tratamento e uma de AF.

Os resultados foram analisados qualitativamente, sendo apresentados em quadros referentes às seguintes análises: do inventário fonético; do sistema fonológico; dos traços distintivos e das generalizações, sendo estas para itens não utilizados no tratamento, para outra posição na palavra, dentro de uma mesma classe de sons e para outras classes de sons.

As crianças selecionadas não apresentaram alterações significativas nas avaliações fonoaudiológicas e complementares, exceto na avaliação fonológica. Na avaliação do sistema estomatognático, foram investigados aspectos referentes às estruturas e funções do sistema estomatognático. Nenhum sujeito apresentou alteração orgânica que limitasse a produção da fala.

No exame articulatório, realizado com o objetivo de analisar a capacidade articulatória da criança por meio da imitação 
retardada de palavras, contendo os fonemas do Português Brasileiro, observou-se que as crianças mantinham as mesmas alterações apresentadas na AFC.

Em uma avaliação de linguagem complementar foi solicitado às crianças que colocassem em ordem cronológica de acontecimentos e depois, contassem uma história baseada em três figuras apresentadas, observando a adequação das respostas, execução de ordens solicitadas, organização lógica do pensamento e estrutura gramatical das sentenças.

A avaliação de vocabulário baseou-se no teste $\mathrm{ABFW}$ e foi realizada com o objetivo de verificar a competência lexical da criança por meio da nomeação de figuras pertencentes a nove campos conceituais: vestuário, animais, alimentos, meios de transporte, móveis e utensílios, profissões, locais, formas e cores, brinquedos e instrumentos musicais.

$\mathrm{Na}$ avaliação psicomotora, foram observados aspectos do desenvolvimento psicomotor de acordo com a idade cronológica, sendo avaliadas a coordenação visomotora, a coordenação dinâmica geral, o controle postural e a organização perceptiva.

A avaliação da discriminação auditiva foi realizada com figuras a fim de avaliar a capacidade da criança em discriminar os fonemas quanto ao ponto, modo de articulação e sonoridade.

A avaliação da CF, realizada com o objetivo de analisar a capacidade da criança em refletir sobre os sons da fala e sua organização na formação das palavras, foi observado dificuldade apenas no nível de fonema.

$\mathrm{Na}$ avaliação da memória, as crianças conseguiram repetir pseudo-palavras de até cinco sílabas, não demonstrando dificuldade de memorizar informações fonológicas.

$\mathrm{Na}$ avaliação simplificada do processamento auditivo, realizada com o objetivo de verificar se a criança apresentava análise metacognitiva dos eventos sonoros, observou-se que as crianças possuíam uma boa compreensão dos eventos sonoros verbais e não-verbais.

Os resultados das avaliações fonológicas serão descritos detalhadamente a seguir. No Quadro 1 estão apresentados o inventário fonético inicial (pré-tratamento) e final (póstratamento) dos três sujeitos da pesquisa.

O S1, 6:4, com DMS apresentava, na avaliação inicial (AI), ausentes no inventário fonético /k/, /l/, /R/ e /L/. No pós-tratamento o inventário fonético estava completo. O S2,
6:0, com DMM apresentava inventário fonético completo na AI, e o manteve no final da terapia. Da mesma forma, S3, 6:0, com DM, apresentava, na AI, inventário fonético completo e o manteve após o tratamento.

No Quadro 2 estão apresentados os dados do sistema fonológico inicial (pré-tratamento) e final (pós-tratamento) dos três sujeitos estudados.

No sistema fonológico do S1, encontravam-se ausentes oito sons (/k/, /g/, /s/, /z/, /l/, /L/, /R/ e /r/) na AI e, após terapia com os pares $/ \mathrm{R} / \mathrm{x} / \mathrm{l} / \mathrm{em} \mathrm{OI}$, seis sons foram adquiridos (/s/, /z/, /z/, /l/, /R/ e /r/), permanecendo ausentes apenas o /r/ em CM. Quanto à posição na palavra, verificou-se um total de oito sons adquiridos após o tratamento, sendo quatro em OI (/s/, /z/, /l/ e /R/), dois em OM (/l/ e /3/) um em CM (/s/) e um em CF $(/ \mathrm{r} /)$.

No sistema fonológico do S2, encontravam-se ausentes cinco sons (/b/, /g/, /v/, /z/ e /3/) na AI e, após terapia com os pares $/ \mathrm{b} / \mathrm{x} / 3 / \mathrm{em} \mathrm{OM}$, foram adquiridos três sons $(/ \mathrm{z} /, / 3 / \mathrm{e}$ $/ \mathrm{r} /$ ), permanecendo ausentes em OI três sons $(/ \mathrm{b} /, / \mathrm{v} / \mathrm{e} / 3 /)$, e em $\mathrm{OM}$ dois sons (/d/ e /g/). Quanto à posição na palavra, verificou-se um total de três sons adquiridos após o tratamento, sendo um na posição de OI (/z/), um em OM (///); e um em $\mathrm{CM}(/ \mathrm{r} /)$.

No sistema fonológico do S3, encontrava-se ausente um som (/n/) na AI e, após terapia com os pares $/ \mathrm{n} / \mathrm{x} / \mathrm{r} / \mathrm{em} \mathrm{OM}$, foram adquiridos três sons $(/ 3 /, / \mathrm{n} / \mathrm{e} / \mathrm{r} /)$. Quanto à posição na palavra, verificou-se um total de quatro sons adquiridos após o tratamento, sendo três na posição de $\mathrm{OM}(/ \mathrm{n} /, / 3 /$ e $/ \mathrm{r} /)$ e um em CM (/r/).

No Quadro 3 estão os traços distintivos que os sujeitos apresentavam alterações no início do tratamento, o tipo de estímulo escolhido para terapia, se 'contraste' ou 'reforço' e os traços alterados pós-terapia.

Para o S1, abordado pelo 'contraste', selecionou-se segmentos-alvo (/R/ x /l/) que contrastavam os traços [dors/ cor+ant], [ \pm cont] e reforçavam o [-voc]. Após o tratamento, dos nove sons não adquiridos na AI, seis foram adquiridos pelo S1. O tratamento por meio do 'contraste' do traço [ \pm cont] favoreceu a aquisição do traço [+cont] (/s/, /z/, /z/, /R/ e /r/). O contraste do traço [dors/cor+ant] favoreceu a aquisição do traço [cor+ant] (/s/, /z/ e /l/).

Para o S2, abordado pelo 'reforço', selecionou-se segmentos-alvo $(/ \mathrm{b} / \mathrm{x} / 3 /)$ que reforçavam os traços [-voc], [+voz]

Quadro 1. Segmentos presentes e ausentes do inventário fonético dos três sujeitos na avaliação inicial (pré-tratamento) e final (pós-tratamento)

\begin{tabular}{|c|c|c|c|c|c|c|c|c|c|c|c|c|c|c|c|c|c|c|c|c|}
\hline \multicolumn{21}{|c|}{ Inventário fonético } \\
\hline Sujeitos & Avaliação & $\mathrm{p}$ & b & $\mathrm{t}$ & d & k & $g$ & $f$ & v & s & z & S & 3 & $\mathrm{~m}$ & $\mathrm{n}$ & $\mathrm{N}$ & I & $r$ & $\mathrm{R}$ & L \\
\hline \multirow{2}{*}{ S1 } & $\mathrm{Al}$ & $\mathrm{p}$ & $\mathrm{b}$ & $\mathrm{t}$ & d & $\varnothing$ & g & $f$ & v & s & z & S & 3 & $\mathrm{~m}$ & $\mathrm{n}$ & $\mathrm{N}$ & $\varnothing$ & $r$ & $\varnothing$ & $\varnothing$ \\
\hline & AF & $\mathrm{p}$ & $\mathrm{b}$ & $\mathrm{t}$ & d & $\mathrm{k}$ & g & $f$ & $\mathrm{v}$ & $\mathrm{s}$ & z & $\mathrm{S}$ & 3 & $\mathrm{~m}$ & $\mathrm{n}$ & $\mathrm{N}$ & 1 & $r$ & $\mathrm{R}$ & $\mathrm{L}$ \\
\hline \multirow{2}{*}{ S2 } & Al & $\mathrm{p}$ & b & $\mathrm{t}$ & d & k & $g$ & $f$ & v & s & z & S & 3 & $\mathrm{~m}$ & $\mathrm{n}$ & $\mathrm{N}$ & 1 & r & $\mathrm{R}$ & L \\
\hline & AF & $\mathrm{p}$ & b & $\mathrm{t}$ & d & k & $g$ & $f$ & v & S & z & S & 3 & $\mathrm{~m}$ & $\mathrm{n}$ & $\mathrm{N}$ & 1 & r & $\mathrm{R}$ & L \\
\hline \multirow{2}{*}{ S3 } & Al & $\mathrm{p}$ & $\mathrm{b}$ & $\mathrm{t}$ & d & k & g & $f$ & $\mathrm{~V}$ & $\mathrm{~s}$ & z & S & 3 & $\mathrm{~m}$ & $n$ & $\mathrm{~N}$ & 1 & $r$ & $\mathrm{R}$ & L \\
\hline & $A F$ & $p$ & b & $t$ & $d$ & k & $\mathrm{g}$ & $f$ & V & $\mathrm{s}$ & z & S & 3 & $\mathrm{~m}$ & $\mathrm{n}$ & $\mathrm{N}$ & I & $r$ & $R$ & L \\
\hline
\end{tabular}

Legenda: ø: sons ausentes no inventário fonético; Al: Avaliação inicial; AF: Avaliação final 
Quadro 2. Sistema fonológico apresentado pelos três sujeitos na avaliação inicial (pré-tratamento) e final (pós-tratamento)

\begin{tabular}{|c|c|c|c|c|c|c|c|c|}
\hline \multirow{2}{*}{ Sujeitos } & \multirow{2}{*}{\multicolumn{3}{|c|}{$\frac{\text { Avaliação inicial }}{\text { Sistema fonológico }}$}} & \multirow{3}{*}{$\begin{array}{l}\text { Alvos de } \\
\text { tratamento }\end{array}$} & \multirow{2}{*}{\multicolumn{4}{|c|}{$\frac{\text { Avaliação final }}{\text { Sistema fonológico }}$}} \\
\hline & & & & & & & & \\
\hline \multirow{5}{*}{ S1 } & Posição & SPA & SNA & & SPA & SNA & SA & $N^{\circ}$ AS \\
\hline & Ol & $/ \mathrm{s} /$ & $/ \mathrm{k} /, / \mathrm{g} /, \mathrm{z} /, \mathrm{l} / \mathrm{l}, \mathrm{R} /$ & \multirow{4}{*}{$\begin{array}{c}/ \mathrm{R} / \mathrm{x} / \mathrm{I} / \\
\mathrm{OI}\end{array}$} & $/ \mathrm{k} /, / \mathrm{g} /$ & & $\begin{array}{l}/ \mathrm{s} /, / \mathrm{z} / \\
/ \mathrm{I} / \mathrm{R} /\end{array}$ & 4 \\
\hline & OM & $|3|$ & $/ \mathrm{k} /, / \mathrm{g} /, \mathrm{Il} /, \mathrm{r} /, \mathrm{L} /, \mathrm{R} /$ & & $\begin{array}{c}/ \mathrm{k} /, / \mathrm{g} /, / \mathrm{L} / \\
/ \mathrm{R} /, / \mathrm{r} /\end{array}$ & & $/ / /, / 3 /$ & 2 \\
\hline & $\mathrm{CM}$ & & $/ \mathrm{r} /, / \mathrm{s} /$ & & & $\mid r /$ & /s/ & 1 \\
\hline & CF & & $\mid r /$ & & & & $/ r /$ & 1 \\
\hline \multirow{4}{*}{$\mathrm{S} 2$} & OI & & /b/, /g/, /z/, /v/, /3/ & \multirow{4}{*}{$/ \mathrm{b} / \mathrm{x} / 3 / \mathrm{OM}$} & $/ g /$ & $/ \mathrm{b} /, / 3 /, / \mathrm{v} /$ & $|z|$ & 1 \\
\hline & OM & $|\mathrm{d} /,| \mathrm{z} \mid$ & /b/, /g/, /v/, /3/ & & $/ \mathrm{b} /, / \mathrm{z} /$ & $/ \mathrm{d} /, / \mathrm{g} /, / \mathrm{v} /$ & $|3|$ & 1 \\
\hline & $\mathrm{CM}$ & $/ \mathrm{r} /$ & & & & & $/ r /$ & 1 \\
\hline & $\mathrm{CF}$ & & & & & & & \\
\hline \multirow{4}{*}{ S3 } & OI & & & \multirow{4}{*}{$\begin{array}{l}/ \mathrm{n} / \mathrm{x} / \mathrm{r} / \\
\mathrm{OM}\end{array}$} & & & & \\
\hline & OM & $|z /, / r|$ & $/ \mathrm{n} /$ & & $/ r /$ & & $/ \mathrm{n} /, / 3 /, / \mathrm{r} /$ & 2 \\
\hline & $\mathrm{CM}$ & $/ r /$ & & & & & $/ r /$ & 1 \\
\hline & CF & & & & $/ r /$ & & & \\
\hline
\end{tabular}

Legenda: OI: Onset inicial; OM: Onset medial; CM: Coda medial; CF: Coda final; SNA: Som não adquirido; SPA: Som parcialmente adquirido; SA: Som adquirido

Quadro 3. Distribuição dos sujeitos conforme os traços distintivos alterados pré e pós-tratamento

\begin{tabular}{|c|c|c|c|c|c|}
\hline Sujeitos & $\begin{array}{l}\text { Traços alterados } \\
\text { pré-tratamento }\end{array}$ & $\begin{array}{l}\text { Segmentos-alvo } \\
\text { selecionados }\end{array}$ & $\begin{array}{c}\text { Traços distintivos } \\
\text { trabalhados }\end{array}$ & $\begin{array}{l}\text { Tipo de estímulo } \\
\text { selecionado }\end{array}$ & $\begin{array}{l}\text { Traços alterados } \\
\text { pós-tratamento }\end{array}$ \\
\hline $\mathrm{S} 1$ & $\begin{array}{c}{[\text {-voc }]} \\
{[\text { dors }]} \\
{[+ \text {-cont }][\text { cor/tant }]}\end{array}$ & $/ \mathrm{R} / \mathrm{x} / \mathrm{l} /$ & $\begin{array}{c}\text { Contrasta } \\
\text { [dors/cor] } \\
\text { [ } \pm \text { cont] } \\
\text { Reforça } \\
\text { [-voc] }\end{array}$ & "contraste" & $\begin{array}{l}\text { [dors }] \\
{[- \text { voc }]}\end{array}$ \\
\hline $\mathrm{S} 2$ & [+voz] & $/ \mathrm{b} / \times / 3 /$ & 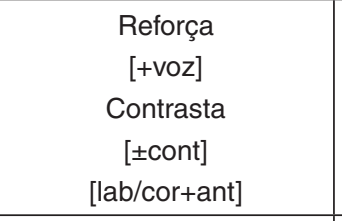 & "reforço" & [+voz] \\
\hline S3 & 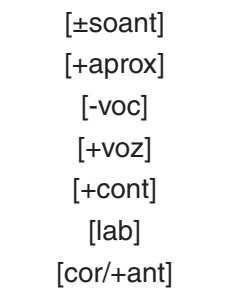 & $/ \mathrm{n} / \mathrm{x} / \mathrm{r} /$ & $\begin{array}{c}\text { Reforça [+soant }] \\
{[\text {-voc }]} \\
{[+ \text { voz }][\text { cor/tant }] \text { Contrasta }} \\
{[ \pm \text { aprox }]} \\
{[ \pm \text { cont }]}\end{array}$ & "reforço" & [-voc] \\
\hline
\end{tabular}

e contrastavam [lab/cor-ant]. Após o tratamento, dos sete sons não adquiridos na $\mathrm{AI}$, três foram adquiridos pelo $\mathrm{S} 2$. O tratamento por meio do 'reforço' do traço [+voz] favoreceu a aquisição desse traço (/z/,/3/ e /r/). Apesar das aquisições apresentadas por $\mathrm{S} 2$, ainda persistiu a dificuldade com o traço [+voz].

O S3 tratado com o par $/ \mathrm{n} / \mathrm{e} / \mathrm{r} /$, contrastavam os traços [+aprox $] /[+$ cont] e reforçavam os traços [+soant]/[-voc]/ [+voz] $/[$ cor/+ant]. Após o tratamento, os três sons não adquiridos na $\mathrm{AI}$, foram adquiridos pelo $\mathrm{S} 3$. O tratamento por meio do reforço do traço [+voz] favoreceu a aquisição desse $\operatorname{traço~}(/ 3 /, / \mathrm{n} / \mathrm{e} / \mathrm{r} /)$.
No Quadro 4 estão apresentadas as generalizações estruturais analisadas a partir do tratamento realizado. Foram analisados os seguintes tipos de generalização: itens não utilizados no tratamento; para outra posição na palavra; dentro de uma mesma classe de sons e para outras classes de sons.

Em relação à generalização para outras palavras que possuíam os sons-alvo, observou-se, para os três sujeitos estudados, que a generalização do S1 foi maior, pois /1/ passou de SNA $(0 \%)$ para SA $(81,81 \%)$ e /R/ de SNA $(0 \%)$ para SPA $(75 \%)$. Para S2 os segmentos permaneceram não estabelecidos, obtendo-se um percentual de produção menor que $40 \%$. E para o S3, o /n/ em palavras não-alvo passou de SNA $(35,29 \%)$ 
Quadro 4. Generalização estrutural para itens não utilizados no tratamento, para outra posição na palavra, para dentro de uma mesma classe de sons e para outra classe de sons

\begin{tabular}{|c|c|c|c|c|c|c|c|c|c|c|c|c|c|}
\hline \multirow[t]{3}{*}{ Sujeitos } & \multirow{3}{*}{$\begin{array}{l}\text { Segmento } \\
\text { tratado }\end{array}$} & \multirow{2}{*}{\multicolumn{2}{|c|}{$\begin{array}{c}\text { Para itens não } \\
\begin{array}{c}\text { utilizados no } \\
\text { tratamento }\end{array} \\
\% \text { Acertos }\end{array}$}} & \multirow{2}{*}{\multicolumn{2}{|c|}{$\begin{array}{c}\begin{array}{c}\text { Outra posição na } \\
\text { palavra }\end{array} \\
\text { \% Acertos }\end{array}$}} & \multicolumn{4}{|c|}{$\begin{array}{l}\text { Dentro de uma mesma } \\
\text { classe de sons }\end{array}$} & \multicolumn{4}{|c|}{ Para outras classes de sons } \\
\hline & & & & & & \multirow[b]{2}{*}{$\mathrm{CE}$} & \multirow[b]{2}{*}{ SNE } & \multicolumn{2}{|c|}{$\%$ Acertos } & \multirow[b]{2}{*}{ CNE } & \multirow[b]{2}{*}{ SNE } & \multicolumn{2}{|c|}{$\%$ Acertos } \\
\hline & & pré & pós & pré & pós & & & pré & pós & & & pré & pós \\
\hline \multirow{4}{*}{ S1 } & \multirow[b]{2}{*}{$\mathrm{R}$} & \multirow{2}{*}{0} & \multirow{2}{*}{75} & \multirow{2}{*}{$0(\mathrm{OM})$} & \multirow{2}{*}{$66,66(\mathrm{OM})$} & \multirow[b]{4}{*}{ Líq } & \multirow[b]{4}{*}{$\kappa$} & \multirow[b]{4}{*}{0} & \multirow[b]{4}{*}{50} & \multirow{2}{*}{ Fric } & s & 78,94 & 100 \\
\hline & & & & & & & & & & & $z$ & 72,72 & 100 \\
\hline & \multirow{2}{*}{ I } & \multirow{2}{*}{0} & \multirow{2}{*}{81,81} & \multirow{2}{*}{$0(\mathrm{OM})$} & \multirow{2}{*}{80 (OM) } & & & & & \multirow{2}{*}{ Plo } & k & 4,54 & 66,66 \\
\hline & & & & & & & & & & & g & 12,5 & 54,54 \\
\hline \multirow{4}{*}{ S2 } & \multirow{2}{*}{$\mathrm{b}$} & \multirow{2}{*}{24,13} & \multirow{2}{*}{28,17} & \multirow{2}{*}{$\mid 16,66(\mathrm{OI})$} & \multirow{2}{*}{$0(\mathrm{OI})$} & \multirow{2}{*}{ Plo } & d & 69,69 & 83,33 & & & & \\
\hline & & & & & & & g & 23,80 & 33,33 & líg & $r$ & 6666 & 100 \\
\hline & & 9 & 3333 & 125 (م) & 20 (ו) & Fric & z & 44,44 & 83,33 & पाप & 1 & 00,00 & 100 \\
\hline & 3 & 9 & 00,00 & I2,5 (Ui) & $20(U 1)$ & rilc & $\mathrm{v}$ & 31,25 & 33,33 & & & & \\
\hline & $\mathrm{n}$ & 35,29 & 90,47 & $83,33(\mathrm{OI})$ & 100 (OI) & Nas & * & * & * & & & & \\
\hline S3 & $r$ & 78,94 & 83,33 & $\begin{array}{c}40(\mathrm{CM}) \\
100(\mathrm{CF})\end{array}$ & $\begin{array}{c}100(\mathrm{CM}) \\
66,66(\mathrm{CF})\end{array}$ & Líq & * & * & * & Fric & 3 & 75 & 100 \\
\hline
\end{tabular}

Legenda: SNE: Segmento não estimulado; OI: Onset inicial; OM: Onset medial; CM: Coda medial; CF: Coda final; CE: Classe estimulada; CNE: Classe não-estimulada; * não houve possibilidade de generalização nesse item

para SA $(90,47 \%)$ e o /r/ de SPA $(78,94 \%)$ para SA $(83,33 \%)$, apresentando uma diferença percentual menor que $\mathrm{S} 1$.

$\mathrm{Na}$ generalização para outra posição na palavra, foram considerados para o S1 os fonemas /R/ e /1/ em OM; para S2, /b/ e /3/ em OI; e, para S3, /n/ em OI e /r/ em CM e CF. Foi possível observar maior generalização em $\mathrm{S} 1$ na posição de OM, sendo que /R/ passou de SNA (0\%) para SPA $(66,66 \%)$ e /l/ de SNA (0\%) para SA (80\%). Em S2 /b/ e /3/ permaneceram SNA em OI. Em S3 /n/ permaneceu SA em OI; /r/ passou de SPA $(40 \%)$ para SA (100\%) em CM e de SA (100\%) para SPA $(66,66 \%)$ em CF.

A generalização dentro de uma mesma classe de sons foi analisada no Quadro 4. Os sons-alvo selecionados para o tratamento do $\mathrm{S} 1$ pertenciam à classe das líquidas, e verificou-se generalização nesta classe com /L/ $(0 \% \rightarrow 50 \%)$ aumentando em 50\% sua porcentagem de ocorrência. Para o S2 foram selecionados sons-alvo envolvendo plosiva e fricativa. Em relação à classe das plosivas, ocorreu generalização para o /d/ $(69,69 \% \rightarrow 83,33 \%)$ aumentando sua porcentagem de ocorrência em $13,64 \%$ e $/ g /(23,80 \% \rightarrow 33,33 \%)$ aumentando sua porcentagem de ocorrência em 9,53\%. Em relação à classe das fricativas, ocorreu generalização para o /z/ $(44,44 \% \rightarrow 83,33 \%)$ aumentando sua porcentagem de ocorrência em $38,89 \%$, e /v/ $(31,25 \% \rightarrow 33,33 \%)$ aumentando sua porcentagem de ocorrência em $2,08 \%$. No tratamento do S3 foram selecionados alvos envolvendo a classe das líquidas e nasais. A generalização não pode ser observada para este caso, pois estavam ausentes apenas os sons-alvo selecionados para tratamento.

Na generalização para outras classes de sons, o S1 apresentou generalização para a classe das fricativas e das plosivas. Na classe das fricativas /s/ passou de SPA $(78,94 \%)$ para SA (100\%) e /z/ de SPA $(72,72 \%)$ para SA (100\%). Na classe das plosivas /k/ passou de SNA $(4,54 \%)$ para SPA $(66,66 \%)$ e /g/ de SNA $(12,50)$ para SPA $(54,54 \%)$. O S2 apresentou generalização para a classe das líquidas, sendo que o /r/ passou de SPA $(66,66 \%)$ para SA (100\%). O S3 apresentou generalização para a classe das fricativas passando o/z/ de SPA (75\%) para SA (100\%).

\section{DISCUSSÃO}

O S1 (DMS) apresentava o maior número de sons ausentes no seu inventário fonético, sendo o sujeito que adquiriu o maior número de sons pós-tratamento (Quadro 1). Isto sugere que existe relação entre o grau de severidade do DF e o número de sons ausentes no inventário fonético pré e pós-tratamento, indicando que quanto mais severo é o DF, maior é a melhora no inventário fonético/fonológico.

Em relação ao número de segmentos adquiridos no sistema fonológico geral pós-tratamento (Quadro 2), S1 (DMS) adquiriu maior número de sons que S2 (DMM), e este maior número de sons que $\mathrm{S} 3(\mathrm{DM})$, concordando com os achados bibliográficos ${ }^{(8)}$, no qual o sujeito com DMS adquiriu seis segmentos, os sujeitos com DMM adquiriram três segmentos e o os dois sujeitos com DM adquiriram um e dois segmentos respectivamente.

$\mathrm{Na}$ análise por traços distintivos (Quadro 3), baseada nas substituições que S1 realizava, e na análise fonológica inicial, observou-se que este apresentava dificuldade com os traços: [-voc], [dors], [ \pm cont] e [cor/+-ant], sendo que a maior parte das substituições envolvia o traço [dors]. Os segmentos-alvo selecionados (/R/e /l/), diferiam em dois traços distintivos que o sujeito apresentava dificuldade ([dors/cor] e [ \pm cont $]$ ), sendo, nesse caso, trabalhado o 'contraste' dos traços.

Os sons-alvo utilizados no tratamento de S1, tratavam-se de dois fonemas novos, com diferença de traços de classe não-principal e com distinção máxima de traços (nível 2), de acordo com a hierarquia de mudanças fonológicas ${ }^{(9)}$. Em estudo $^{(8)}$ utilizando a abordagem pelo 'contraste', por meio do 
uso de segmentos situados no nível 2, foi possível observar a ocorrência de generalizações.

A análise por traços distintivos, baseada nas substituições que S2 realizava e na análise fonológica inicial, mostrou que este apresentava dificuldade com os traços: [-voc], [+voz] e [cor/-ant], sendo que a maior parte das substituições envolvia o traço [+voz]. Os segmentos-alvo selecionados (/b/e /3/) reforçavam o traço distintivo em que o sujeito apresentava dificuldade ([+voz]), apesar de também contrastarem [lab] e [cor+ant].

Os sons-alvo utilizados no tratamento de S2, tratavam-se de dois fonemas novos, com diferença de traços de classe não-principal e com distinção máxima de traços (nível 2), de acordo com a hierarquia de mudanças fonológicas ${ }^{(9)}$.

$\mathrm{Na}$ análise por traços distintivos, baseada nas substituições que S3 realizava e, na análise fonológica inicial, mostrou que este apresentava dificuldade com os traços: [-voc], [+/-soant], [+aprox], [+voz], [lab], [+cont] e [cor/+ant], sendo que a maior parte das substituições envolvia o traço [-aprox]. Os segmentos-alvo selecionados $(/ \mathrm{n} / \mathrm{e} / \mathrm{r} /)$, reforçam quatro traços distintivos que o sujeito apresentava dificuldade ([cor/+ant], [+soant], [-voc] e [+voz]).

Os sons-alvo utilizados no tratamento de $\mathrm{S} 3$, tratavam-se de um fonema novo, com distinção de traços de classe principal e com distinção máxima de traços (nível 2), de acordo com a hierarquia de mudanças fonológicas ${ }^{(9)}$. Os segmentos $/ \mathrm{n} /, / \mathrm{z} / \mathrm{e} / \mathrm{r} /$ em OM e /r/ em CM foram adquiridos. Já /r/ em CF estava SA na AI e passou para SPA na AF. Um decréscimo no desempenho lingüístico em certos momentos do desenvolvimento pode ocorre por haver, em um dado momento, um desenvolvimento mais acentuado de um nível lingüístico em detrimento de outro ${ }^{(1)}$.

Em relação à generalização para itens não utilizados no tratamento, isto é, palavras que continham o som-alvo, mas que não foram diretamente usados na terapia pôde ser observada nos três sujeitos estudados. Em um estudo de caso $^{(13)} \mathrm{de}$ um sujeito, com DMM e idade de cinco anos, tratado com o /R/ em OI e OM pelo modelo ABAB-Retirada e Provas Múltiplas, foram observadas generalizações a outras palavras não utilizadas no tratamento. Esse tipo de generalização também foi encontrado em outros estudos ${ }^{(6,8,14)}$.

Em relação às generalizações para outra posição na palavra, neste item foi analisada a generalização para outras posições na palavra, diferentes daquelas utilizadas como alvo de terapia, sendo observada nos três sujeitos estudados. Estudo relatou esse tipo de generalização em falantes do Inglês ${ }^{(14)} \mathrm{e}$ do Português Brasileiro ${ }^{(6,8)}$.

Em relação às generalizações dentro de uma classe de som, foi analisada a generalização para outros fonemas pertencentes à mesma classe de sons daqueles utilizados como alvo. Apenas, no S3 a generalização não pode ser observada para este caso, pois estavam ausentes apenas os sons-alvo selecionados para tratamento. Em um estudo ${ }^{(13)}$ não se observou a generalização do som-alvo dentro de uma classe de sons, concordando com o resultado do S3; entretanto, outros estudos verificaram a ocorrência desse tipo de generalização ${ }^{(6,14)}$.

Em relação às generalizações para outras classes de sons, foi observada a generalização a outras classes diferentes das utilizadas como alvo, sendo encontrada nos três sujeitos estudados, concordando com os achados dos estudos ${ }^{(8,14)}$ realizados com falantes do português, os quais relataram a generalização para outras classes de sons. Assim como estudos com falantes do Inglês ${ }^{(14)}$ encontraram esse tipo de generalização.

\section{COMENTÁRIOS FINAIS}

Ao final deste estudo, foi possível apresentar as seguintes conclusões: tanto os sujeitos tratados pelo 'contraste', como os sujeitos tratados pelo 'reforço', apresentaram mudanças favoráveis em seus sistemas fonológicos, tendo sido o S1 o que obteve maior número de segmentos adquiridos, entretanto, não se pode afirmar que o tratamento pelo 'contraste' foi o único responsável, pelo fato do grau do DF dos sujeitos serem diferentes. Sugere-se a realização de estudos com tratamento pelo 'contraste' ou 'reforço' utilizando um único traço para análise de outros resultados, bem como com sujeitos com o mesmo grau de severidade do desvio fonológico.

Quanto à generalização, observou-se que os três sujeitos tiveram resultados favoráveis, pois ocorreu generalização para itens não-utilizados no tratamento, para outra posição na palavra, dentro de uma mesma classe de sons e para outras classes de sons.

\begin{abstract}
The aim of this study was to verify the phonological changes obtained with treatment by the Modified Maximal Oppositions Model, using "reinforcement" or "contrast" approach, in subjects with different severity levels of phonological disorders (PD). Three male children, mean age six years old, with different severity levels of PD were submitted to speech and language evaluations, including phonological assessment and complementary exams. After initial evaluation, they were treated by the Modified Maximal Oppositions Model, using the "reinforcement" or "contrast" approach to distinctive features. The following structural generalizations were verified: items that were not used in the treatment; another position in the word; inside the same sound class; and another sound class. After the therapeutic process, subjects' phonetic inventories were complete, but the phonological system was incomplete for all subjects. S1, treated using the contrast [dors/cor] and [+/-cont], was still facing difficulty with [dors] and [-voc] after treatment. S2, treated based on reinforcement with [+voice], had a persistent difficulty regarding [+voice] after treatment. S3, treated by the reinforcement with [+soant], [-voc], [+voice] and [cor/+ant], showed, after treatment, difficulty only with [-voc]. After the therapeutic process, all types of structural generalizations were observed. It was found that the subjects treated by "reinforcement" as well as the one treated by "contrast" presented generalizations. As a result, it was not possible to conclude which was the most efficient approach to the treatment of PD.
\end{abstract}

Keywords: Speech; Articulation disorders; Language; Child 


\section{REFERÊNCIAS}

1. Lamprecht RR. (org.) Aquisição fonológica do português: perfil de desenvolvimento e subsídios para a terapia. Porto Alegre: Artmed; 2004.

2. Shriberg LD, Kwiatkowski J. Phonological disorders I: a diagnostic classification system. J Speech Hear Disord. 1982;47(3):226-41.

3. Bagetti T, Mota HB, Keske-Soares M. Modelo de oposições máximas modificado: uma proposta de tratamento para o desvio fonológico. Rev Soc Bras Fonoaudiol. 2005;10(1):36-42.

4. Gierut JA. Maximal opposition approach to phonological treatment. J Speech Hear Disord. 1989;54(1):9-19.

5. Keske-Soares M, Mota HB, Pagliarin KC, Ceron MI. Estudo sobre ambientes favoráveis à produção da líquida não-lateral /r/ no tratamento do desvio fonológico. Rev Soc Bras Fonoaudiol. 2007;12(1):48-54.

6. Pereira LF, Mota HB. Tratamento fonológico baseado nos contrastes de oposições máximas. Pró-Fono. 2002; 14(2):165-74.

7. Pagan LO, Wertzner HF. Intervenção no distúrbio fonológico por meio dos pares mínimos com oposição máxima. Pró-Fono. 2002;14(3):31324.
8. Bagetti T. Mudanças fonológicas em sujeitos com diferentes graus de severidade do desvio fonológico tratados pelo modelo de oposições máximas modificado [dissertação]. Santa Maria: Universidade Federal de Santa Maria; 2005

9. Gierut JA. The conditions and course of clinically induced phonological change. J Speech Hear Res. 1992;35(5):1049-63.

10. Yavas M, Hernandorena CM, Lamprecht RR. Avaliação fonológica da criança. Reeducação e terapia. Porto Alegre: Artes Médicas; 1991.

11. Bernhardt B. Developmental implications of nonlinear phonological theory. Clin Linguist Phon. 1992;6(4):259-81.

12. Keske-Soares M. Terapia fonoaudiológica fundamentada na hierarquia implicacional dos traços distintivos aplicada em crianças com desvios fonológicos [tese]. Porto Alegre: Pontifícia Universidade Católica do Rio Grande do Sul; 2001.

13. Barberena LS, Keske-Soares M, Mota HB. Generalização no tratamento com o /R/ em um caso de desvio fonológico médio-moderado. Rev Soc Bras Fonoaudiol. 2004;9(4):229-36.

14. Powell TW, Elbert M. Generalization following the remediation of early- and later-developing consonant clusters. J Speech Hear Disord. $1984 ; 49(2): 211-8$ 